\title{
Publisher Correction: A mass spectrometry-based proteome map of drug action in lung cancer cell lines
}

Benjamin Ruprecht (D), Julie Di Bernardo, Zhao Wang, Xuan Mo D, Oleg Ursu, Matthew Christopher, Rafael B. Fernandez, Li Zheng, Brian D. Dill D, Huijun Wang, Yuting Xu DD, Andy Liaw, Jonathan D. Mortison, Nirodhini Siriwardana,

Brian Andresen, Meir Glick, James R. Tata, Victoria Kutilek, Ivan Cornella-Taracido and An Chi (iD)

Correction to: Nature Chemical Biology https://doi.org/10.1038/s41589-020-0572-3, published online 20 July 2020

In the version of this article originally published, the file for 'Supplementary Data 1' was omitted, and its entry in the Supplementary Information list was incorrectly linked to the file for 'Supplementary Data 5'. The error has been corrected in the HTML version of the paper.

Published online: 11 August 2020

https://doi.org/10.1038/s41589-020-0643-5

(๑) The Author(s), under exclusive licence to Springer Nature America, Inc. 2020 